1 Closing Gaps But Increasing Bias In North American Butterfly Inventory Completeness

2 Authors: Vaughn Shirey', Michael W. Belitz², Vijay Barve², Robert Guralnick ${ }^{2}$

3 ' Department of Biology, Georgetown University; Washington, DC USA

$4 \quad{ }^{2}$ Florida Museum of Natural History, University of Florida, Gainesville, FL USA

5

6 Corresponding author:

7 Vaughn Shirey, vms55@georgetown.edu; ORCID: 0000-0002-3589-9699

8 Box 571229 Reiss Science Bldg., Room 406, 37th and O Streets, NW Washington DC 20057

9

10 Additional authors:

11 Michael W. Belitz, ORCID: 0000-0002-8162-5998

12 Vijay Barve, ORCID: 0000-0002-4852-2567

13 Robert Guralnick, ORCID: 0000-0001-6682-1504

14

15 Statement of authorship: VS, MWB, VB, and RG developed the initial research idea. VS, MWB, 16 and VB curated the data and VS performed the analysis. VS, MWB, VB, and RG wrote and edited

17 the manuscript together.

19 Funding: VS was supported by Georgetown University. MWB was supported by a University of

20 Florida Biodiversity Institute Fellowship. VB and RG were supported by the ButterflyNet project

21 (DEB-1541500).

\title{
23 Conflicts of Interest
}

24 The authors report no conflicts of interest. 


\section{Acknowledgements}

27 We would like to thank the countless museum staff and community scientists for their tremendous

28 work in digitizing and documenting butterfly records from across the continent. We appreciate

29 Michelle Duong and the Map of Life informatics team for help with Mexican distribution data.

30 Map of Life provides visualizations of range products utilized here.

Data Deposition: Scripts and author generated data are stored both within a Github repository

33 cited in the text as well as a static Zenodo archive. Other, $3^{\text {rd }}$ party datasets are cited throughout

34 the text.

Abstract: Aggregate biodiversity data from museum specimens and community observations have

37 promise for macroscale ecological analyses. Despite this, many groups are under-sampled, and

38 sampling is not homogeneous across space. Here we used butterflies, the best documented group

39 of insects, to examine inventory completeness across North America. We separated digitally

40 accessible butterfly records into those from natural history collections and burgeoning community

41 science observations to determine if these data sources have differential spatio-taxonomic

42 biases. When we combined all data, we found startling under-sampling in regions with the most

43 dramatic trajectories of climate change and across biomes. We also found support for the

44 hypothesis that community science observations are filling more gaps in sampling but are more

45 biased towards areas with the highest human footprint. Finally, we found that both types of

46 occurrences have familial-level taxonomic completeness biases, in contrast to the hypothesis of

47 less taxonomic bias in natural history collections data. These results suggest that higher inventory

48 completeness, driven by rapid growth of community science observations, is partially offset by 
bioRxiv preprint doi: https://doi.org/10.1101/2020.07.20.212381; this version posted July 22, 2020. The copyright holder for this preprint (which

was not certified by peer review) is the author/funder, who has granted bioRxiv a license to display the preprint in perpetuity. It is made available under aCC-BY 4.0 International license.

49 higher spatio-taxonomic biases. We use the findings here to provide recommendations on how to

50 alleviate some of these gaps in the context of prioritizing global change research.

51

52 Keywords: butterflies, sampling bias, sampling completeness, GBIF, north America, global

53 change

54

55

56 


\section{INTRODUCTION}

The mobilization of openly and freely available natural history data has increased the

59 ability for researchers to access information about species distribution and abundance in a given

60 time and place. In recent years, these data have been augmented by community science programs

61 which facilitate collection of biodiversity observations and digital vouchers from a network of

62 volunteers. Aggregated data from both natural history collections and community science

63 programs have been used to answer often broad questions in ecology, including assessing

64 extinction risks for understudied groups (Carlson et al. 2017, Seppälä et al. 2018) and modeling

65 species response to environmental change (Eskildsen et al. 2015).

Despite the utility of these data, many taxa are still under-sampled (Troudet et al. 2017)

67 and prevalent biases in the spatiotemporal distribution of these data are noteworthy (Beck et al.

68 2013, Meyer et al. 2015). These biases imply that inventory completeness (how many species have

69 been recorded vs. how many are expected to occur) is uneven across time and space. Given the

70 urgency to understand ecological responses to many global change processes, knowing where

71 sampling has and has not occurred to a sufficient degree is critical for both prioritizing effort to

72 close information gaps and choosing extents and scales for macroecological analyses. The

73 enormous growth of community science reporting for some groups promises to rapidly close

74 inventory gaps, but less is known about how specimens from natural history collections and

75 community science data may be differentially spatially biased. Community science volunteers may

76 stay closer to developed areas to sample biodiversity than collectors who may be more attentive to

77 collecting in under-sampled regions. This may lead to larger under-sampling by community

78 scientists in remote regions, including the far North, which is projected to experience the most 
79 dramatic effects of climate change. Under-sampling in the Arctic and other sparsely populated

80 regions negatively impacts ability to assess how climate has impacted communities over time.

81 Butterflies (Lepidoptera: Papilionoideae) are a diverse group of organisms that are

82 relatively less sampled compared to vertebrate fauna (Troudet et al. 2017) which have been the

83 focus on previous sampling completeness assessments (Meyer et al. 2015). Additionally,

84 butterflies have been widely used to detect signals of global change (Parmesan et al. 1999,

85 Eskildsen et al. 2015). Given the value of butterflies as an indicator group, we aim to test how well

86 sampled North America is for butterflies using natural history collections and community science

87 data, as gaps in openly accessible biodiversity data limit efforts to address ecological, evolutionary,

88 and conservation questions. More specifically, we utilize estimates of distributions from field

89 guides to establish a baseline richness value at multiple, coarse scales usable for presence

90 prediction (Jetz et al. 2012). We then compare that value to richness derived from occurrence

91 records from the Global Biodiversity Information Facility (GBIF), iDigBio, and eButterfly.

92 We separated occurrence records into those from natural history collections and from

93 community science-based observations and examined temporal trends in the number of records

94 and completeness for each. We then tested the hypothesis that both types of occurrences were

95 biased to areas where the humans are likely to be most active, but that those biases were

96 particularly severe for community science records. We also examine if there are differences across

97 butterfly families among these record types, presuming that records from natural history

98 collections are less likely to be biased in familial completeness coverage. To provide further

99 context for these results, we ask how biomes and climate regimes are sampled differently in order

100 to provide meaningful information for global change ecologists and other users of these data. 
101 Finally, we discuss potential strategies to mitigate under-sampling across the continent in the 102 future.

\section{MATERIAL AND METHODS}

Occurrence records were obtained from GBIF (GBIF 2020), iDigBio (iDigBio 2020), and eButterfly (Prudic et al. 2017) from 1950 through 2019 within North America (Canada, Mexico,

107 United States). Range maps of species found in the United States and Canada were digitized from 108 the Kaufman Field Guide to Butterflies of North America (Brock and Kaufman 2006). For species 109 found in Mexico, range maps were digitized from A Swift Guide to Butterflies of Mexico and 110 Central America (Glassberg 2018) as part of the ButterflyNet project, which are digitally available

111 for visualization on Map of Life (Jetz et al. 2012). These maps only include known source 112 population locations, and do not include distributions of strays. All range maps from the sources

113 were merged into a single shapefile consisting of many spatial polygons which were clipped to 114 only terrestrial areas within North America. These range maps were then intersected with 115 continent-wide equal area grids at $100 \mathrm{~km}, 200 \mathrm{~km}$, and $400 \mathrm{~km}$ resolution. A species was 116 considered to occupy a $100 \mathrm{~km}$ cell if its range passed within $2,000 \mathrm{~m}$ of the grid centroid and

117 considered to occupy a cell at coarser resolutions if it intersected the grid cell irrespective of 118 distance to the cell centroid. Taxonomic names across the fishnet grids and occurrence data were 119 harmonized to a single taxonomic list using R package taxotools (Barve 2020) and the small 120 minority of names that could not be resolved manually after the process were discarded from the 121 analysis. We analyzed only occurrence records that fell within the boundaries of their species' 122 range map but recorded how many records fell outside of these boundaries over time to assess any 123 potential temporal degradation of range maps. 
125 within a grid cell to the number of overlapping range maps within that grid cell. In some cases due

126 to range map exclusion along coastlines and because we only included species present in this

127 fishnet if it occurred within $2000 \mathrm{~m}$ of the grid centroid, this ratio was slightly higher than 1.0 and

128 was thus floored to 1.0. The occurrence dataset was then filtered by the basis of the record, year,

129 and taxonomy attributes to examine how specimen-only (listed as preserved specimen or material

130 sample), community observation-only (listed as human observations from the basisOfRecord field

131 in Darwin Core), time period, and the taxon-rank of family (which are monophyletic, Espeland et

132 al., 2018) impacted completeness scores. Machine observations were a small fraction of these data

133 and were not included in the analysis.

134 Overall average completion between specimen and observation data was assessed using a

135 t-test. We then tested average completion differences among families using an ANOVA on the

136 combined, specimen, and community observation datasets, and differences in the number of cells

137 complete at or over $50 \%$ using a Chi-square test for families between specimen and observation

138 based datasets. Post-hoc testing was conducted with Bonferroni correction in the case of Chi139 square.

140 We also assembled spatial data including velocity of climate under RCP 8.5 forecasts into 1412085 (AdaptWest 2015); human footprint, representing areas where there are built environments, 142 roads, or converted land (Venter et al. 2016); protected regions (Dept. of Forestry and Natural 143 Resources, Clemson University for CEC 2010); and biomes as designated by the World Wildlife 144 Fund (Olson et al. 2001). For human footprint and climate velocity, we calculated average values, 145 and for protected areas, the percent coverage of those areas, within each $100 \mathrm{~km}$ grid cell. For 146 biome type, we determined the majority biome within each $100 \mathrm{~km}$ grid. We used these resampled 
147 values alongside the completion scores to identify drivers of sampling completeness and under-

148 sampled regions described in more detail below.

For potential drivers of completeness, we considered human footprint and protected areas

150 to each represent areas where humans may be actively reporting butterfly occurrences, and

151 specified separate linear models for the combined, museum specimen, and community observation

152 datasets as (Sampling Completeness $\sim$ Human Footprint + Protected Region Cell Coverage). We

153 also ran these univariate models using either human footprint or protected areas as predictors.

154 Model selection was then performed using AIC as the selection criterion to determine the top

155 model. We compared model goodness of fit for the best models for natural history versus

156 community science in order to assess the differential impact these factors may have on datasets

157 with potentially different underlying observation strategies.

Finally, we examined the sampling completeness within the cells with the most extreme

$15910 \%$ and $25 \%$ of climate velocities and the sampling completeness across the WWF biomes found

160 in North America. We removed from our analysis biomes in which the number of $100 \times 100 \mathrm{~km}$

161 cells was less than 10. This included mangrove forests, tropical grasslands, and flooded

162 grasslands. All data preparation and analysis was performed in R version 3.6.3 "Holding the

163 Windsock" (R Core Team 2020) using the packages tidyverse, sp, sf, raster, data.table, mapdata,

164 maptools, gridExtra, stringr, rgdal, ggforce, exactextractr, and scales (Pebesma et al. 2005,

165 Auguie 2017, Brownrigg 2018, Pebesma 2018, Dowle and Srinivasan 2019, Pedersen 2019,

166 Wickham 2019a, Wickham 2019b, Baston 2020, Bivand 2020, Bivand and Lewin-Koh 2020,

167 Hijmans 2020, Wickham and Seidel 2020). The script utilized here is available from a public

168 GitHub repository at [anonymized]. It is also available with our generated datasets via a Zenodo

169 archive at [anonymized]. 


\section{RESULTS}

We obtained approximately 2.8 million records from our aggregate iDigBio, GBIF, and

173 eButterfly datasets. Overall, $91.2 \%$ of occurrence records fell within range map delineations for

174 their respective species. This has changed little over time with an average annual percentage of $17588.6 \%$ from 1950-2019 and a recent increase within the last decade of sampling to 91.4\%. From 1761950 to 2019 , the ratio of cells sampled biyearly at $80 \%$ completion by museum specimen data to 177 those by community observations alone decreased dramatically, especially in the last decade of 178 sampling with community based completion becoming more prevalent as the number of 179 community observations increases (Figure 1).

\section{$\underline{\text { Human Footprint and Protected Areas }}$}

In all cases, the best performing model according to AIC included human footprint alone

183 without the percentage of protected natural areas (Table 1). For museum records, the variance

184 explained by the model was low $\left(\mathrm{R}^{2}=0.09\right)$ compared to the composite dataset $\left(\mathrm{R}_{2}^{2}=0.25\right)$ and the

185 community science dataset $\left(\mathrm{R}^{2}=0.29\right)$.

187 Geographic and Taxonomic Sampling Completeness

Sampling completeness was not homogeneous across scales with noticeable geographic

189 gaps in the far north, midwest, and northern Mexico as illustrated in Figure 2. Mean specimen and 190 observation-based completeness was significantly different according to our t-test (-13.27, 2919

191 DF, $\mathrm{p}<0.0001)$, with observations having a higher average completion ratio $(0.40+/-0.007 \mathrm{SE}$

192 to $0.27+/-0.006 \mathrm{SE}$ ). Sampling was also inconsistent across families, especially within the 
193 Lycaenidae. To illustrate this better, in the composite dataset, differences among completeness

194 across families were significant according to $\operatorname{ANOVA}\left(\mathrm{F}_{(4,726)}=51.49, \mathrm{p}<0.0001\right)$ (Figure $\left.3 \mathrm{a}\right)$ and

195 ANOVA also supported significant differences across families for the specimen based $\left(\mathrm{F}_{4,}\right.$

$\left.196{ }_{5336)}=86.44, \mathrm{p}<0.0001\right)($ Figure $3 \mathrm{~b})$ and observation based $\left(\mathrm{F}_{(4,6325)}=44.72, \mathrm{p}<0.0001\right)($ Figure $3 \mathrm{c})$

197 datasets (Post-hoc test results in Supplemental Figure 1). Chi-square tests to assess differences in

198 the number of $100 \times 100 \mathrm{~km}$ cells completed at $50 \%$ or more between specimens and observations

199 revealed there is a significant association with family-level completion and basis of record

$200\left(\mathrm{X}^{2}=31.04,4 \mathrm{DF}, \mathrm{p}<0.0001\right)($ Figure 3b,c). Post hoc comparisons (Beasley and Schumacker 1995)

201 revealed that this association was significant for Nymphalidae and Pieridae with observations

202 having more cells at $50 \%$ or more complete in these families $(\mathrm{p}<0.01)$ (Figure $3 \mathrm{~d})$.

\section{Sampling in Projected Novel Climate Regimes and Biomes}

Of the 80 th and 95 th percentile $100 \mathrm{~km}$ resolution grid cells experiencing the most dramatic

206 climate effects on average under RCP 8.5 into $2080,97.5 \%$ and $97.2 \%$ fell below the $80 \%$

207 sampling completeness mark respectively, indicating under-sampling in these regions (Figure 4).

208 In addition, sampling across biomes at the $100 \times 100 \mathrm{~km}$ resolution was inconsistent, with some

209 biomes being sampled on average more completely than others as illustrated in Figure 5. Only the

210 Mediterranean woodland/scrub biome demonstrated over 80\% sampling completeness on average

211 with notable under-sampling occurring in deserts, tropical, and boreal/arctic regions. Moderate

212 sampling (between 50\% - 80\% completeness on average) was demonstrated within most mid-

213 latitude temperate regions. 


\section{DISCUSSION}

Sample completeness across North American has accelerated in recent years, driven

218 strongly by the growing number of community observations generated from programs such as

219 iNaturalist (observations cited in our GBIF download), who share research grade observations with

220 GBIF, and eButterfly (Figure 1a). The majority of cells with $>80 \%$ completeness are now from

221 community science data, which continues to grow exponentially per year (Figure 1b),

222 demonstrating the importance of these data for closing distribution knowledge gaps into the future.

223 A large volume of community science records may be due to the ease of submission. For example,

224 iNaturalist submissions can be completed by simply taking a photograph on a mobile phone.

225 Networks such as eButterfly often appeal more directly to dedicated amateur lepidopterists, and

226 do not require photo vouchers to publish data, which has the potential to allow for more

227 observations of butterfly species that are difficult to photograph. This is in contrast to specimen-

228 based data in which preparation, curation, and digitization are all required steps to publish 229 occurrence data.

Despite this influx of community science data, sampling is still inconsistent across space

231 and taxonomy (Figure 2, 3). Regions with low human footprint are frequently under-sampled or

232 not sampled at all, and our simple model validates this finding alongside other studies that have

233 examined the relationship between human population densities and record densities (Girardello et

234 al. 2019). A key finding is that these biases towards sampling where human infrastructure is the

235 most developed are stronger for community observation data than for specimens (Table 1). Thus,

236 community science observations are not likely to be a full panacea for closing inventory

237 knowledge gaps. While some areas of North America are likely to be inventoried at increasingly

238 finer spatial grain with burgeoning growth of community science data, other areas may remain 
perniciously under-sampled. This likely continuing butterfly inventory knowledge gap in remote

240 regions is thus both particularly challenging and crucial to overcome since these are exactly the

241 areas forecasted to experience the most climatic change. A particularly good example are polar

242 regions of North America, where climate velocities are often particularly high (Figure 4 shows the

243 80th and 95th percentile of highest velocities) and sampling is woefully incomplete. (Figure 4).

244 As well, even some mid and low-latitude biomes are under-sampled, including deserts and many

245 tropical biomes in which butterfly diversity is extremely high (Willig et al. 2003) (Figure 3). We

246 argue that community science alone is unlikely to solve existing gaps in biodiversity monitoring

247 unless those programs are directed into sparsely populated regions through socially responsible

248 excursions or other research campaigns that consult with local stakeholders and Indigenous

249 communities. These directed and collaborative efforts, requiring partnerships and coordination,

250 will help to provide a critical basis for mapping and ultimately monitoring butterfly diversity in

251 order to detect changes in the face of shifting climate regimes.

We had anticipated that traits that make some butterflies easier to detect, photograph and

253 identify might be biased across butterfly families, thus leading to familial-level biases in

254 completeness. We expected these issues to be more acute for community scientists, compared to

255 professional collectors, who presumably are collectively more knowledgeable and trained in

256 sampling methods that might reduce bias. We already demonstrated reduced spatial biases for

257 natural history specimen collecting, which might also suggest better sampling of habitats, also

258 potentially reducing taxonomic biases. In the composite dataset, Lycaenidae exhibit lower average

259 completeness with most other groups differing from each other as well (Supp. Figure 1),

260 supporting our hypothesis of taxonomic biases in completeness. However, we did not find

261 evidence that natural history specimen collecting led to less taxonomically biased sampling, at 
least at the familial level. We did however find that completeness from community science

263 observations was higher compared to natural history specimen records only for nymphalids and

264 pierids and not for other butterfly families. While higher completeness itself is not surprising given

265 the trends we report, we had anticipated either similar trends across groups, or that showy groups

266 such as the swallowtail butterflies (i.e. Papilionidae) were more likely to be biased in favor of

267 community science observations given they are generally colorful, large, and charismatic. Further

268 exploration using species-level trait data to tease apart these patterns is warranted. In particular,

269 it may be that species-level rarity may be particularly important, especially if phylogenetically

270 conserved. Other traits that may be worth examining include habitat and flight preferences (canopy

271 vs. understory fliers) that directly relate to ease of human observation.

272 Our study expands upon prior work done on butterfly inventory completeness (Girardello

273 et al. 2019) by including an independent baseline richness via digitized maps at coarse resolution

274 and by examining the contributions of specimens and community observations. In addition, with

275 a narrower focus on just North America and by including an assessment of sampling completeness

276 in regions with high climate velocity and across biomes, we can better assess which areas are in

277 need of targeted sampling in the future. Specifically, and in contrast to previous work (Girardello

278 et al. 2019), we found a severe lack of sampling in the most northern regions of North America.

279 This urgency to sample the north is further supported by the stark reality that these regions are also

280 experiencing the most drastic impacts of climate change (Manabe and Stouffer 1980, Gauthier et

281 al. 2015). Overall, several key regions should be prioritized for sampling including: (a) tundra and

282 boreal forest, (b) tropical forests, and (c) deserts. Given the relatively low human population

283 densities of these regions, funding directed towards establishing community science initiatives,

284 and partnerships among organizations with interests in butterfly monitoring, will likely be critical 
alongside complementing these initiatives with specimen collection and focal digitization of

286 records in these regions.

\section{Conclusions}

289 Butterfly inventory completeness is not uniform across North America. Our research has

290 revealed continuing under-sampling in regions that are facing threats from climate change as

291 well as within specific biomes across the continent. Additionally, family level differences in

292 sampling completeness may be driven by species traits and abundance, leading to disparities in

293 completeness across taxa. In order to mitigate some of these issues, attention should be drawn

294 towards establishing community partnerships of both opportunistic and structured survey

295 systems in under-sampled regions. It is clear that community science provides a strong

296 mechanism for alleviating sampling shortfalls and has potential to provide finer-grained views of

297 butterfly communities, but only if such initiatives are also directed farther from regions with the

298 densest human populations and travel infrastructure. Furthermore, additional curation and

299 digitization of museum specimens will be critical in developing a historical backbone for

300 analyses across time and space. Millions of specimens still remain undigitized in arthropod

301 natural history collections (Cobb et al. 2019), and the continuation of funding for museum staff

302 and biodiversity informatics infrastructure will be critical in mobilizing these data needed for

303 ecological research, especially potential for some kinds of temporal trend analyses (Soroye et al.

304 2020). Supporting digitization in tandem with concerted efforts to direct community science

305 initiatives towards under-sampled regions will move us towards unlocking the full potential of

306 these opportunistic data in an era of global change. 


\section{References}

309

310

AdaptWest Project. 2015. Gridded climate velocity data for North America at $1 \mathrm{~km}$ resolution. Available at adaptwest.databasin.org.

Auguie, B. 2017. GridExtra: Miscellaneous functions for “grid” graphics. R package version 2.3. https://CRAN.R-project.org/package=gridExtra.

Barve, V. 2020. Taxotools: Tools to handle taxonomic lists. R package version 0.0.43. http://doi.org/10.5281/zenodo.3934939.

Baston, D. 2020. Exactextractr: Fast extraction from raster datasets using polygons. R package version 0.3.0. https://CRAN.R-project.org/package=exactextractr.

Beasley, T.M., Schumacher, R.E. 1995. Multiple regression approach to analyzing contingency tables: Post hoc and planned comparison procedures. - The Journal of Experimental Education 64: 79-93.

Beck, J., Ballesteros-Mejia, L., Nagel, P., Kitching, I.J. 2013. Online solutions and the Wallacean shortfall: What does GBIF contribute to our knowledge of species' ranges?. - Diversity and Distributions 19: 1043-1050.

Bivand, R., Keitt, T., Rowlingson, B. 2020. Rgdal: Bindings for 'geospatial' data abstraction library. R package version 1.5-10. https://CRAN.R-project.org/package=rgdal.

Bivand, R., Lewin-Koh, N. 2020. Maptools: Tools for handling spatial objects. R package version 1.0-1. https://CRAN.R-project.org/package=maptools. 
327 Brock, J.P., Kaufman, K. 2006. Kaufman field guide to butterflies of North America. - Houghton Mifflin Harcourt.

329

330
Brownrigg, R. 2018. Mapdata: Extra map databases. R package version 2.3.0. https://CRAN.Rproject.org/package $=$ mapdata

Carlson, C.J., Burgio, K.R., Dougherty, E.R., Phillips, A.J., Bueno, V.M., Clements, C.F., Castaldo, G., Dallas, T.A., Cizauskas, C.A., Cumming, G.S., Doña, J. 2017. PArasite biodiversity faces extinction and redistribution in a changing climate. - Science Advances 3: e1602422.

Cobb, N.S., Gall, L.F., Zaspel, J.M., Dowdy, N.J., McCabe, L.M., Kawahara, A.Y. 2019. ASsessment of North American arthropod collections: Prospects and challenges for addressing biodiversity research. PeerJ 7:e8086.

Dept. of Forestry and Natural Resources, Clemson University for the Commission for Environmental Cooperation. 2010. Terrestrial protected areas of North America, 2010. http://www.cec.org/naatlas.

Dowle, M., Srinivasan, A. 2019. Data.table: Extension of 'data.frame’ R package version 1.12.8. https://CRAN.R-project.org/package=data.table.

Eskildsen, A., Carvalheiro, L.G., Kissling, W.D., Biesmeijer, J.C., Schweiger, O., Нøye, T.T. 2015. Ecological specialization matters: long-term trends in butterfly species richness and assemblage composition depend on multiple functional traits. - Diversity and Distributions 21: 792-802. 
347 Espeland, M., Breinholt, J., Willmott, K.R., Warren, A.D., Vila R., Toussaint, E.F., Maunsell,

348 S.C., Aduse-Poku, K., Talavera, G., Eastwood, R., Jarzyna, M.A. 2018. A comprehensive

349 and dated phylogenomic analysis of butterflies. - Current Biology 28(5): 770-778.

350 Gauthier, S., Bernier, P., Kuuluvainen, T., Shvidenko, A.Z., Schepaschenko, D.G. 2015. Boreal

351 forest health and global change. - Science 349: 819-822.

352 GBIF.org (05 June 2020) GBIF Occurrence Download https://doi.org/10.15468/dl.8v7jwf

353 Girardello, M., Chapman, A., Dennis, R., Kaila, L., Borges, P.A.V., Santangeli, A. 2019. Gaps in

354 butterfly inventory data: A global analysis. - Biological Conservation 236: 289-295.

355 Glassberg, J. 2018. A swift guide to butterflies of Mexico and Central America. - Princeton $356 \quad$ University Press.

357 Hijmans, R.J. 2020. Raster: Geographic data analysis and modeling. R package version 3.1-5. 358 https://CRAN.R-project.org/package=raster.

359 iDigBio (02 April 2020) iDigBio Occurrence Download (records citation file in supplemental $360 \quad$ materials).

361 Jetz, W., McPherson, J.M., Guralnick, R.P. 2012. Integrating biodiversity distribution knowledge: 362 Toward a global map of life. - Trends in Ecology and Evolution 27: 151-159.

363 Manabe, S., Stouffer, R.J. 1980. Sensitivity of a global climate model to an increase of CO2 364 concentration in the atmosphere. - Journal of Geophysical Research: Oceans 85: 55295554. 
Meyer, C., Kreft, H., Guralnick, R., Jetz, W. 2015. Global priorities for an effective information basis of biodiversity distributions. - Nature Communications 6: 1-8.

Olson, D.M., Dinerstein, E., Wikramanayake, E.D., Burgess, N.D., Powell, G.V., Underwood, E.C., D’amico, J.A., Itoua, I., Strand, H.E., Morrison, J.C., Loucks, C.J. 2001. Terrestrial ecoregions of the world: A new map of life on Earth: A new global map of terrestrial ecoregions provides an innovative tool for conserving biodiversity. - Bioscience 51: 933-

Pedersen, T.L. 2019. Ggforce: Accelerating 'ggplot2.' R package version 0.3.1. https://CRAN.Rproject.org/package=ggforce.

Parmesan, C., Ryrholm, N., Stefanescu, C., Hill, J.K., Thomas, C.D., Descimon, H., Huntley, B., Kaila, L., Kullberg, J., Tammaru, T., Tennent, W.J., 1999. Poleward shifts in geographical ranges of butterfly species associated with regional warming. - Nature 399: 579-583.

Pebesma, E.J.. Bivand, R.S. 2005. Classes and methods for spatial data in R. R News 5 (2). https://cran.r-project.org/doc/Rnews/.

Pebesma, E., et al. 2018. Simple Features for R: Standardized Support for Spatial Vector Data. The R Journal 10 (1), 439-446, https://doi.org/10.32614/RJ-2018-009 Insects 8: 53 . 
R Core Team. 2020. R: A language and environment for statistical computing. R Foundation for Statistical Computing, Vienna, Austria.

387

Seppälä, S., Henriques, S., Draney, M.L., Foord, S., Gibbons, A.T., Gomez, L.A., Kariko, S., Malumbres-Olarte, J., Milne, M., Vink, C.J., Cardoso, P. 2018. Species conservation profiles of a random sample of world spiders I: Agelenidae to Filistatidae. - Biodiversity Data Journal 6.

Soroye, P., Newbold, T., Kerr, J. 2020. Climate change contributes to widespread declines among bumble bees across continents. - Science 367(6478): 685-688.

Troudet, J., Grandcolas, P., Blin, A., Vignes-Lebbe, R., Legendre, F. 2017. Taxonomic bias in biodiversity data and societal preferences. - Scientific Reports 7.

Venter, O., Sanderson, E.W., Magrach, A., Allan, J.R., Beher, J., Jones, K.R., Possingham, H.P., Laurance, W.F., Wood, P., Fekete, B.M., Levy, M.A. 2016. Global terrestrial human footprint maps for 1993 and 2009. - Scientific Data 3: 1-10.

Wickham, H., et al. 2019. Welcome to the tidyverse. Journal of Open Source Software, 4(43), 1686, https://doi.org/10.21105/joss.01686.

Wickham, H. 2019. Stringr: Simple, consistent wrappers for common string operations. R package version 1.4.0. https://CRAN.R-project.org/package=stringr.

Wickham, H., Seidel, H. 2020. Scales: Scale functions for visualization. R package version 1.1.1. https://CRAN.R-project.org/package=scales. 
bioRxiv preprint doi: https://doi.org/10.1101/2020.07.20.212381; this version posted July 22, 2020. The copyright holder for this preprint (which

was not certified by peer review) is the author/funder, who has granted bioRxiv a license to display the preprint in perpetuity. It is made available under aCC-BY 4.0 International license.

404 Willig, M.R., Kaufman, D.M., Stevens, R.D. 2003. Latitudinal gradients of biodiversity: Pattern, 405 process, scale, and synthesis. - Annual Review of Ecology, Evolution, and Systematics 34:

406 273-309.

407 
409 Tables

410 Table 1. Coefficient estimates of each multiple regression model for the full record set, specimen-

411 only record set, and community observation recordset. Delta-AIC values indicate the difference

412 between the multiple regression and simple regression model which included only human footprint

413 as a predictor variable. In all cases, models excluding protected area percentage outperformed the

414 simple regression according to AIC.

415

\begin{tabular}{|c|c|c|c|c|c|c|}
\hline & estimate & se & $t$ & p-value & $\boldsymbol{R}^{2}$ & delta-AIC \\
\hline$\underline{\text { All Records }}$ & & & & & 0.25 & 1327.03 \\
\hline Intercept & 0.354 & 0.0083 & 42.63 & $<0.0001$ & & \\
\hline Human Footprint & 0.027 & 0.0011 & 23.92 & $<0.0001$ & & \\
\hline Specimens & & & & & 0.09 & 1160.43 \\
\hline Intercept & 0.194 & 0.0089 & 21.57 & $<0.0001$ & & \\
\hline Human Footprint & 0.014 & 0.0012 & 12.14 & $<0.0001$ & & \\
\hline$\underline{\text { Observations }}$ & & & & & 0.29 & 1144.99 \\
\hline Intercept & 0.238 & 0.0088 & 26.96 & $<0.0001$ & & \\
\hline Human Footprint & 0.028 & 0.0011 & 24.79 & $<0.0001$ & & \\
\hline
\end{tabular}




\section{$418 \quad$ Figures}

(a)

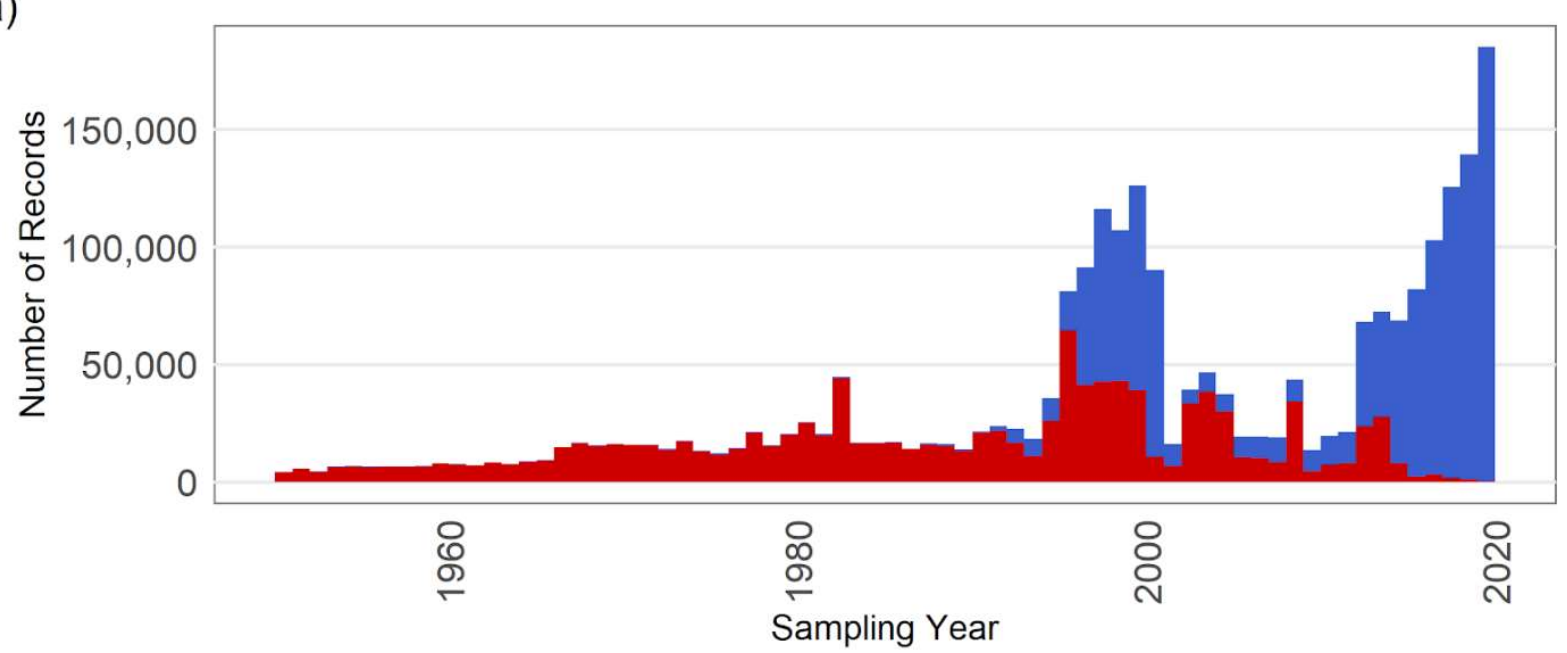

(b)

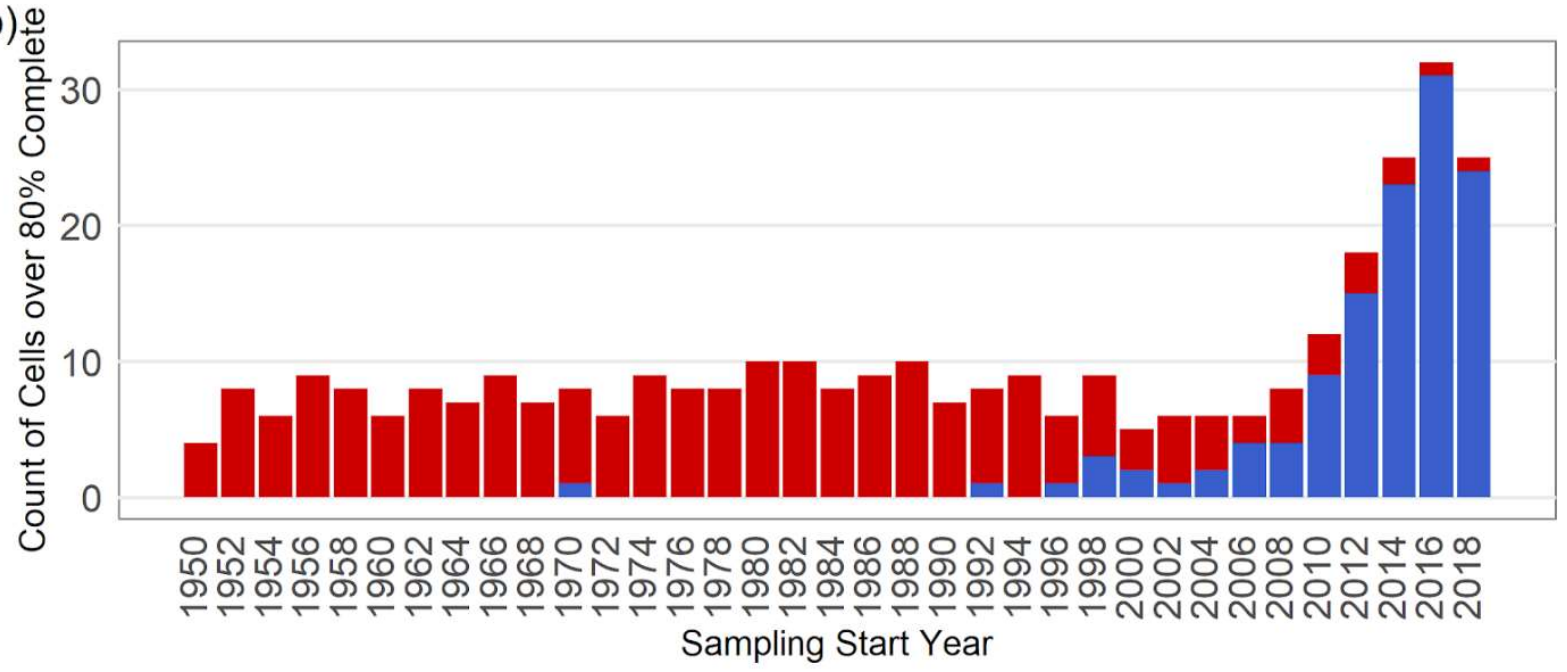

420 Figure 1. (a) The number of museum specimens and community observation-based occurrence

421 records over time, stacked by year. (b) Number of cells at $100 \mathrm{~km}$ resolution that are over $80 \%$

422 complete and that meet that threshold by museum or community observation data alone

423 biyearly. $\mathrm{Red}=$ museum specimens; Blue = community observations. 


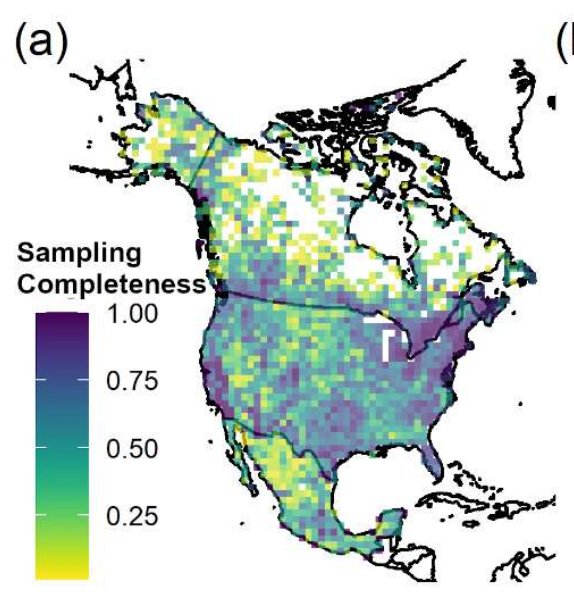

(b)
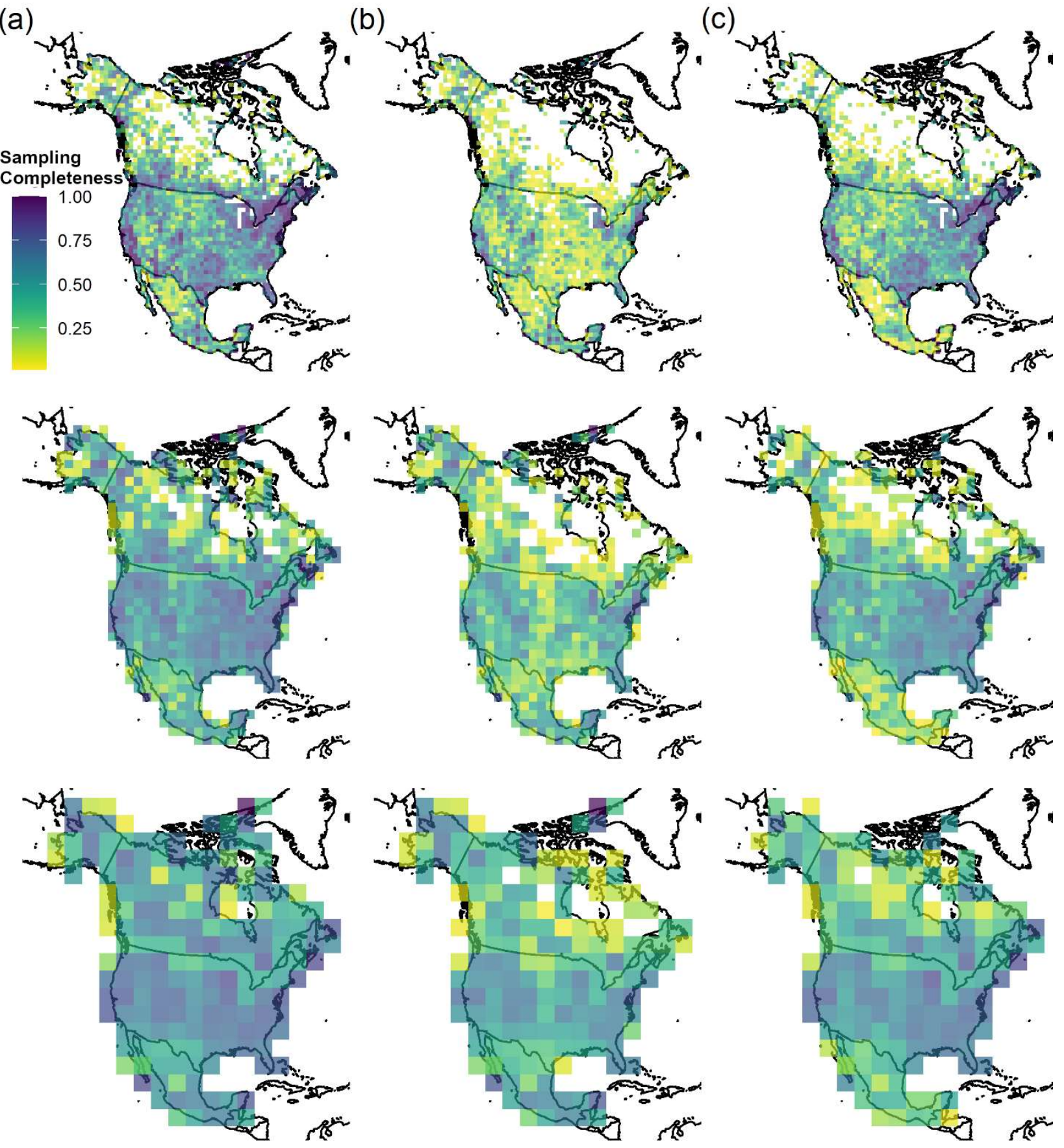

426 Figure 2. Sampling completeness within cells of varying spatial resolution $(100 \mathrm{~km}, 200 \mathrm{~km}$,

427 400km) across North America from 1950-2019 based on record source (a) all records, (b)

428 specimens, and (c) community observations. 
(a)

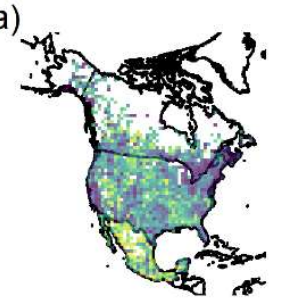

Lycaenidae

Hesperiidae

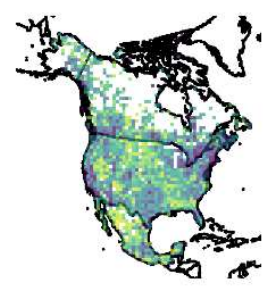

Nymphalidae

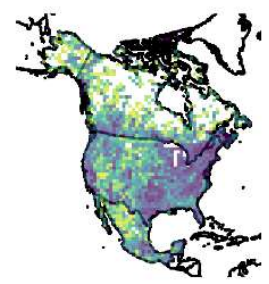

Papilionidae

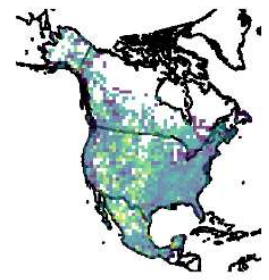

Pieridae

430

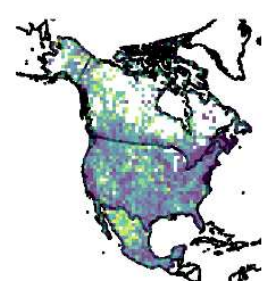

(b)
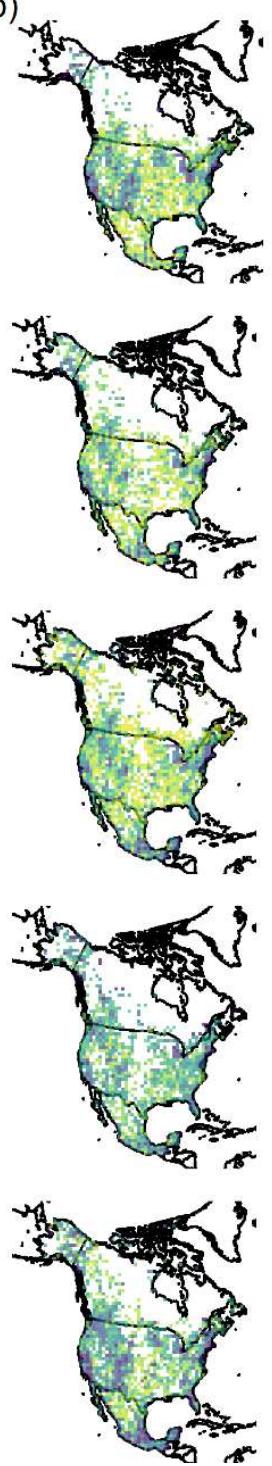

(c)
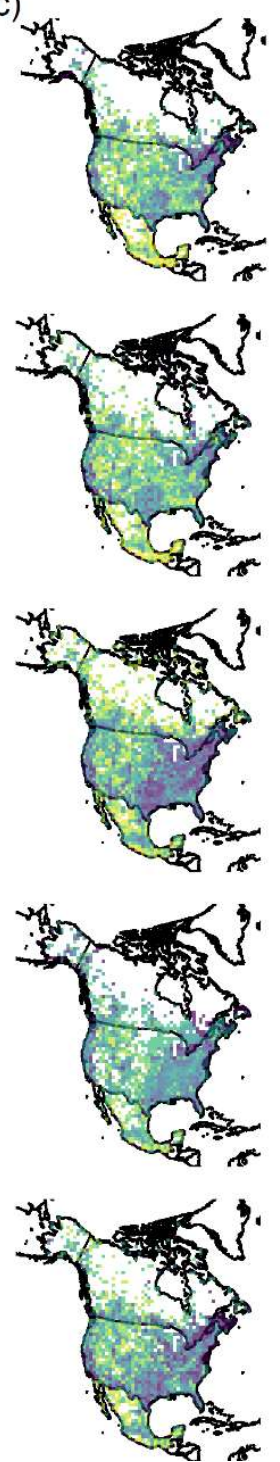

(d)
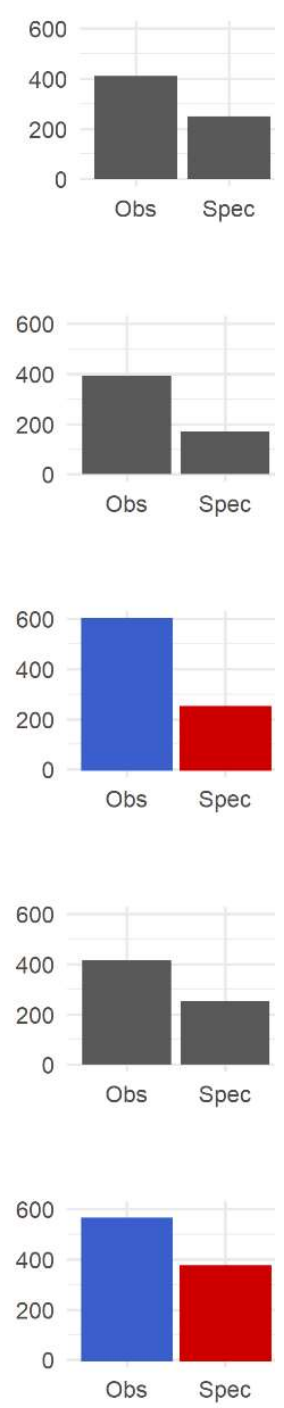

431 Figure 3. Sampling completeness among butterfly families at 100x100km resolution across

432 North America from 1950-2019 by family and by record source (a) all records, (b) specimens,

433 and (c) community observations. Panel (d) illustrates the number of cells over 50\% complete in

434 each family, colored plots indicate a significant contribution to the chi-square statistic after

435 Bonferroni corrected post-hoc tests. 


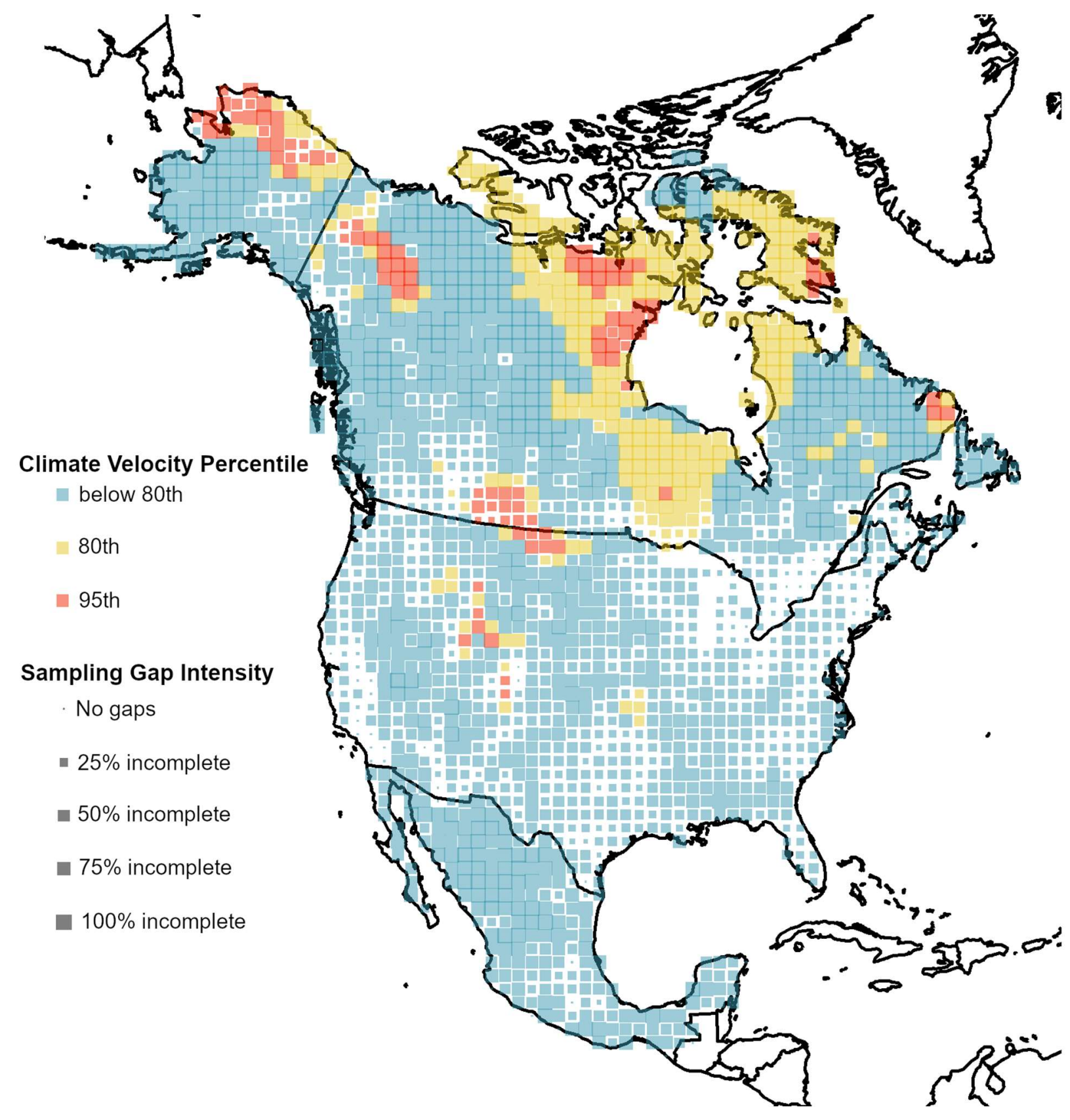

Figure 4. Sampling completeness of 100x100km grid cells by climate velocity percentile. Size of

439 the point within the cell indicates sampling incompleteness (larger cells are less sampled).

440 Yellow and red cells are the 80th and 95th percentile of climate velocities respectively. Blue

441 cells fall underneath the 80th percentile for climate velocity. Climate velocity rasters do not

442 extend into northern Nunavut. 


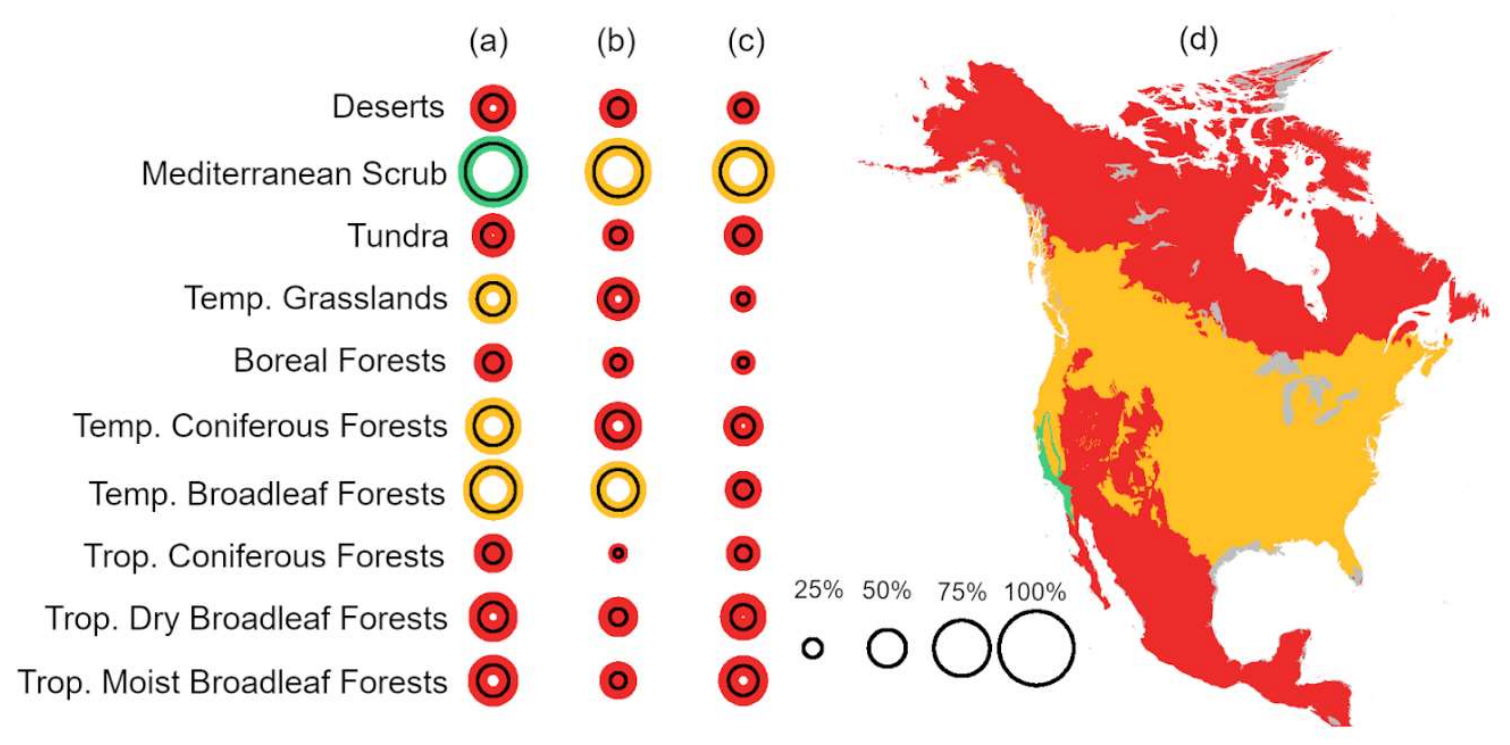

445 Figure 5. Mean +/- SD sampling completeness across WWF biomes, (a) composite dataset, (b)

446 community observations only, and (c) museum specimens only. Panel (d) displays the biomes

447 utilized without delineation for clarity and includes coloration based on average composite

448 sampling completeness. Red $=$ sampling below 50\% average completeness, Yellow $=$ sampling

449 average between $50 \%$ and $80 \%$ completeness, Green $=$ sampling average at or above $80 \%$

450 completeness. Grey regions represent unsampled areas, or regions where the number of

$451100 \times 100 \mathrm{~km}$ cells within a biome was $<15$. 
(a)

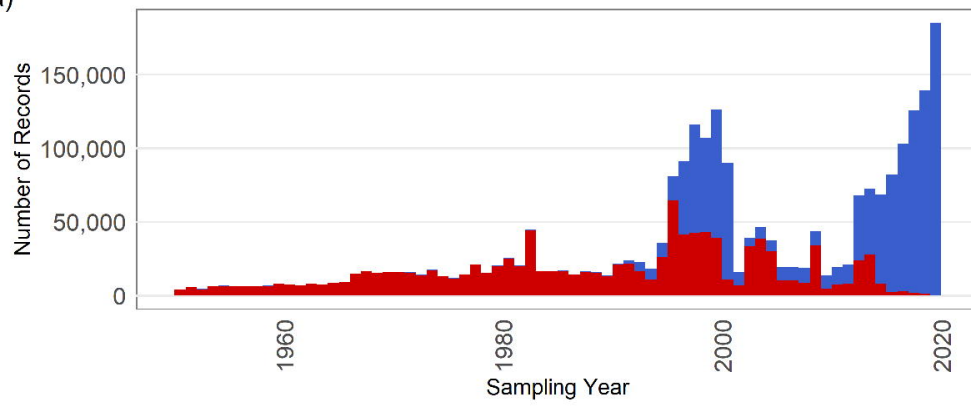

(b) $\frac{\Phi}{\omega}$

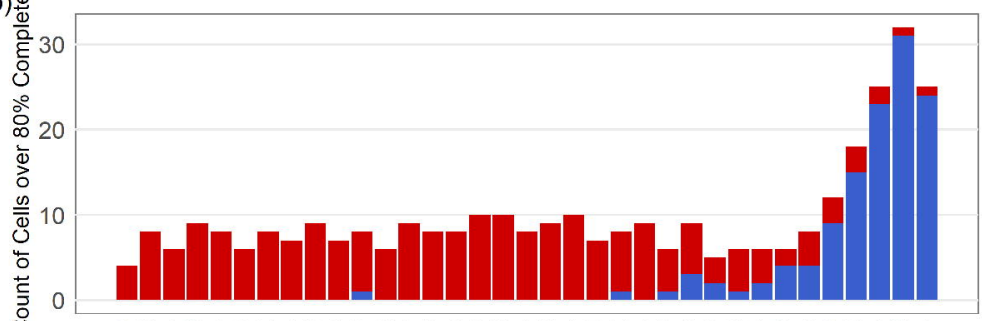

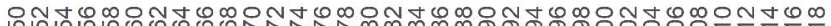

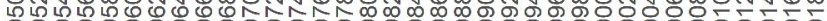

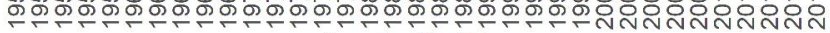


(a)

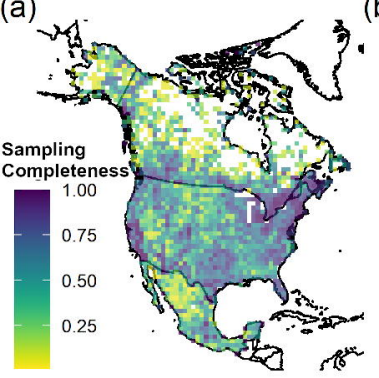

(b)

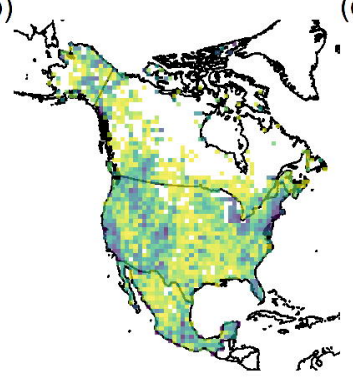

(c)

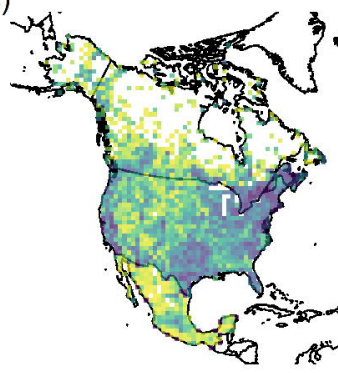

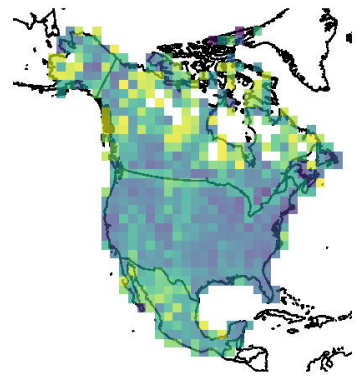
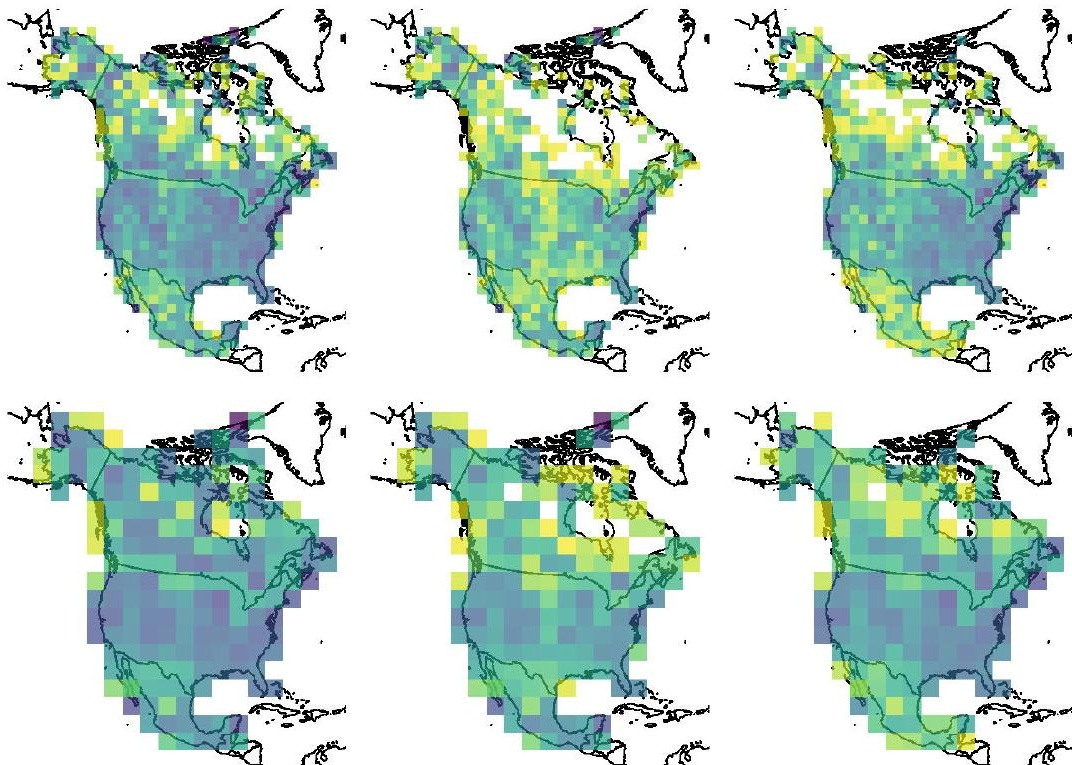
(a)

Lycaenidae

Hesperiidae
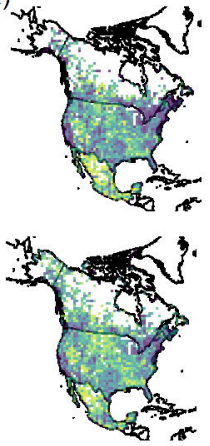

Nymphalidae

Papilionidae
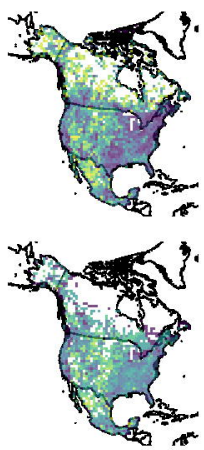

Pieridae (b)

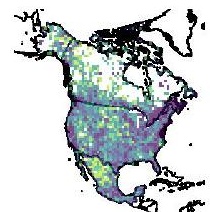

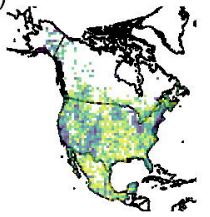
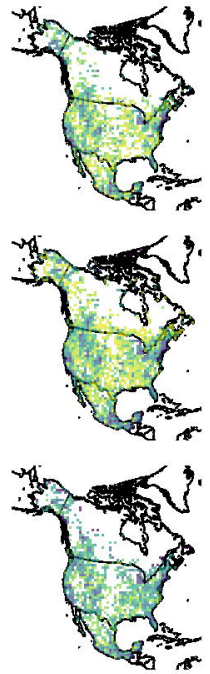

(c)

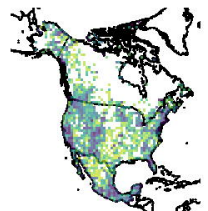

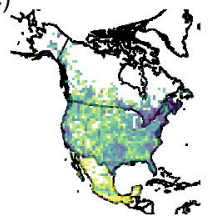
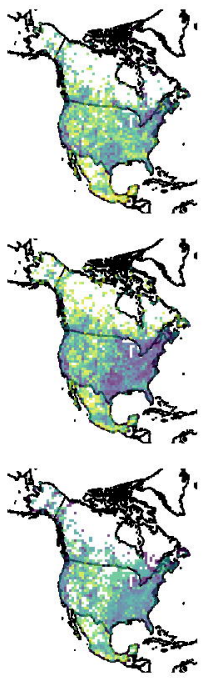

(d)

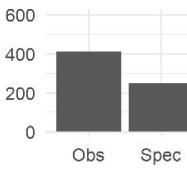

600

400

200

0

Obs

Spec

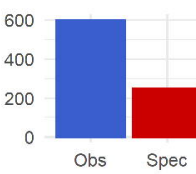

600

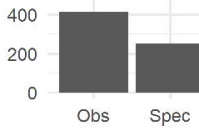

ind

600

400

200

0

Obs

Spec 


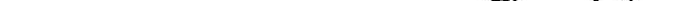


(a) (b) (c)

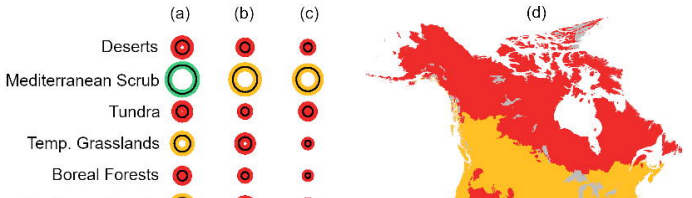

Temp. Coniferous Forests 0 (

Temp. Broadleaf Forests $\mathrm{O} \quad \mathrm{O}$

Trop. Coniferous Forests 0

Trop. Dry Broadleaf Forests

- 0

Trop. Moist Broadleaf Forests

o

o.

等

- 000 